\title{
Upregulation of SHMT2 Promotes Tumor Growth and Poor Prognosis of Breast Cancer through Activating VEGF and MAPK Signaling Pathway
}

\section{Shuang-Yan Xie}

Sun Yat-sen University Cancer Center

Dingbo Shi ( $\nabla$ shidb@sysucc.org.cn )

Sun Yat-sen University Cancer Center

Fei Lin

Sun Yat-sen University Cancer Center

Xiao-Yu Cheng

Sun Yat-sen University Cancer Center

Tong-chao Cheng

Sun Yat-sen University Cancer Center

Yi Ouyang

Sun Yat-sen University Cancer Center

Wen Xia

Sun Yat-sen University Cancer Center

Ling Guo

Sun Yat-sen University Cancer Center

Huan-Xin Lin

Sun Yat-sen University Cancer Center

Research article

Keywords: SHMT2, Breast Cancer, MAPK, VEGF, Serine/glycine metabolism

Posted Date: November 23rd, 2021

DOI: https://doi.org/10.21203/rs.3.rs-1016628/v1

License: (c) (i) This work is licensed under a Creative Commons Attribution 4.0 International License.

Read Full License 


\section{Abstract}

Background: Serine hydroxymethyltransferase 2 (SHMT2) is a key enzyme in Serine/glycine metabolism. SHMT2 is very important for tumor cell growth and proliferation as well as metabolism. Here, we investigated the regulatory effects of SHMT2 on breast cancers growth and identified the underlying mechanisms of functions.

Methods: We detected the expression of SHMT2 in breast cancer cells and tissues by immunohistochemistry and Western blotting. We investigated the functional and molecular mechanisms by which SHMT2 downregulation or overexpression regulates the growth and apoptosis of breast cancer cells in vivo and in vitro.

Results: We found SHMT2 was highly expressed in BRCA cell lines and tumor tissues. Strong SHMT2 expression showed a positive correlation with the poor prognoses of patients with breast cancers. SHMT2 knockdown by shRNA significantly inhibited cell growth and induced apoptosis in vitro, and whereas SHMT2 overexpression promoted tumor growthin in subcutaneous xenograft model. RNA-seq revealed that high expression of SHMT2 not only promoted serine metabolism, nucleotide metabolism, oxidative phosphorylation and proteasome independent degradation pathways. It also activated the cell survival signaling pathway and antagonized the apoptosis pathway. The observed molecular regulation of cell growth was accompanied by the activited of the MAPK, VEGF pathways and suppressed of the mitochondrial mediated apoptosis pathway that was mediated by the SHMT2 up-regulation.

Conclusions: These results indicate that SHMT2 plays a critical role in regulating breast cancers growth and could serve as a therapeutic target for breast cancer therapy.

\section{Introduction}

As the most commonly diagnosed cancer type among women, breast cancer forms a major threat to women's health globally(1). It is mainly divided into the following four molecular subtypes, luminal $A$, luminal B, triple negative, and HER2. The former two subtypes display high expression of estrogen receptor (ER) and progesterone receptor (PR) and changed level of HER2. triple negative subtype displays negative expression of ER, PR and HER2, and the patients with this subtype usually owns poor prognosis. HER2 subtype displays negative expression of ER, PR and high expression of HER2 and other genes associated with proliferation $(2,3)$. Although progresses have been made in understanding breast cancer biology, the molecular mechanisms associated with proliferative regulation of breast cancer cells, the acquisition of invasion and metastasis phenotype, and the development of therapeutic assistance were still poorly understood. Thus, considering the high complexity and variability in breast cancer development, the discovery and identification of new breast cancer biomarkers and therapeutic targets and the mention and application of new therapeutic strategies based on this seems more significant.

Cumulative evidence suggests that the serine and glycine biosynthetic pathways are linked to the biosynthesis of cellular components to sustain cell proliferation. Dysfunction of genes involved in the 
one-carbon unit biosynthetic pathway has been linked to increased risks of various human cancers $(4,5)$.

Serine Hydroxymethyltransferase 2 (SHMT2) has been a research hotspot in recent years. SHMT2 is a PLP-dependent tetrameric enzyme that catalyzes the reversible transition from serine to glycine, thus promoting the production of one-carbon units that are indispensable for cell growth and regulation of the redox and epigenetic states of cells.SHMT2 is a key enzyme in one-carbon unit metabolism and catalyzes the conversion of serine and tetrahydrofolate (THF) to glycine and 5,10methylenetetrahydrofolate(6-8).Under a hypoxic environment, SHMT2 can be upregulated and could promote the generation of nicotinamide adenine dinucleotide phosphate(NADPH) and glutathione for maintaining the redox balance. Accumulating evidence confirmed that SHMT2 facilitates cell proliferation and tumor growth and is tightly associated with poor prognosis $(6,9)$.

In humans, SHMT consists of two isoforms, SHMT1, which is located in the cytosol (cSHMT), and SHMT2, which is located in the mitochondria (mSHMT). These two isoforms display an amino acid sequence identity of approximately $66 \%$. In addition, SHMT1 forms a tetramer in the cytoplasm, while SHMT2 exists in a dimer-to-tetramer transition depending on pyridoxal 5'-phosphate (PLP) binding(10). In fact, SHMT2 can encode SHMT2a, a second transcript that lacks the import signal location to the mitochondria and is localized in the cytoplasm. Due to its important role in the cycle of one-carbon unit, SHMT2 is ubiquitous and highly conserved in all organisms(11). In addition, previous reports have indicated that SHMT2 expression is upregulated in various types of cancer, including leukemia(12),lymphoma(13, 14), rhabdomyosarcoma(15),colorectal cancer(16-18), Lung cancer (19$21)$ and hepatocellular Carcinoma $(22,23)$, and it is correlated with cancer progression and poor prognosis, indicating that SHMT2 is a potential and novel target for the diagnosis and treatment of tumors, reflecting that SHMT2 is partly involved in the process of tumorigenicity. SHMT2 may be an oncogene, SHMT2 could be acetylated at lysine K95 in different cancer cells, including CRC cells. K95 acetylation inhibits SHMT2 enzymatic activity and promotes its degradation via macroautophagy. SIRT3, a deacetylase localized in the mitochondria, deacetylated SHMT2 and eventually increased its activity to promote cell proliferation and colorectal carcinogenesis(17).SHMT2 also interacts with nicotinamide adenine dinucleotide (NAD+)-dependent lysine desuccinylase SIRT 5 directly. Under metabolic stress, desuccinylation at lysine 280 of SHMT2 by SIRT 5 enhances SHMT2 activity to accelerate cell metabolism and facilitate cell proliferation and tumour growth in vivo and in vitro(24).SHMT2 was highly expressed in breast cancer cells, and the expression level of SHMT2 was positively correlated with breast cancer grade(25).Gene set enrichment analysis revealed that SHMT2 was significantly associated with gene signatures of mitochondrial module, cancer invasion, metastasis and may be a valuable prognostic biomarker in ER-negative breast cancer cases $(26,27)$.however, so far, the molecular mechanism of SHMT2 promoting breast cancer growth and metastasis is still not very clear.

In the present study, we detected SHMT2 protein expression in breast cancer cell lines and tumor tissues.We also analyzed breast cancer samples from tissue arrays and evaluated the prognostic value of SHMT2 in breast cancer.The effects of SHMT2 knockdown or overexpression on the growth of breast cancer cells were evaluated and the underlying molecular mechanisms were further elucidated. The role of 
SHMT2 in breast cancer growth was further demonstrated in vivo by breast cancer xenograft models. Our findings provide new insights into the biological role of SHMT2 in breast cancer and suggest that SHMT2 may be a new therapeutic target for breast cancer.

\section{Methods}

\section{Cell lines and cell culture}

Human breast cancer cell lines (HCC1806, MDA-MB-231, MCF-7, ZR-75-1, BT549) and normal breast cell lines (MCF-10A) were obtained from the American Type Culture Collection (ATCC, Manassas, VA). The cells were cultured as monolayers in RPMI-1640 culture medium (Invitrogen, Carlsbad, CA) supplemented with $10 \%$ fetal bovine serum, $100 \mu \mathrm{g} / \mathrm{ml}$ penicillin, and $100 \mu \mathrm{g} / \mathrm{ml}$ streptomycin and maintained in an incubator with a humidified atmosphere of $95 \%$ air and $5 \% \mathrm{CO} 2$ at $37^{\circ} \mathrm{C}$.

\section{Reagents and antibodies}

Antibodies against SHMT2(A1215),GAPDH(A19056), $\beta$-actin(AC026), VEGF(A12303), PEDF(A3475), were obtained from ABclonal Technology Co.,Ltd. (ABclonal,wuhan,China). Antibodies against JNK(AF6318), P38(AF6456), ERK(BF8004), P-JNK(Thr183+Tyr185)(AF3318), P-P38(Thr180/Tyr182) (AF4001), PERK(Thr202/Tyr204)(AF1015), cytochrome-c (AF0146), PARP(DF7198), Cleaved-PARP (Asp214) (AF7023), Cleaved-Caspase 3 (Asp175)(AF7022), Cleaved-Caspase 9 (Asp353) (AF5240), BAX(AF0120), Bcl-2 (AF6139), Goat Anti-Rabbit IgG (H+L) HRP (S0001) and Goat Anti-Mouse IgG (H+L) HRP (S0002) were purchased from Affinity Biosciences (Affbiotech, Jiangshu,China).ERK MAPK inhibitor FR180204 (HY-12275), p38 MAPK inhibitor SB202190 (HY-10295) were purchased from the Med Chem Express(MCE).

\section{Preparation of shRNA or expression plasmid}

The SHMT2 shRNA (HSH109479) and the overexpression plasmid of SHNT2 (p-SHMT2) (T3093) were purchased from GeneCopoeia, Inc. (Guangzhou, China). The sequence of the human SHMT2-specific shRNA was 5'-TCTGAACAACAAGTACTCGG-3' and 5'-TCTCAGGATCACTGTCCGAC-3', and the scramble shRNA was 5'-GGC TCC GAACGGGTCACGATT-3'. For the in vitro delivery of the shRNA and plasmid into tumor cells, the sequences were first encapsulated into Lipofectamine 3000 (L3000150, Thermo Scientific,) that had validated by many analyses by dissolving in Opti-MEM. The knockdown efficiency was validated by western blot.

\section{Transient transfection}


A total of $2 \times 10^{5}$ HCC1806 and ZR-75-1 cells were seeded into each well of a six-well tissue culture plate (Nunc). The next day (when the cells were $70-80 \%$ confluent), the culture medium was aspirated, and the cell monolayer was washed with prewarmed sterile phosphate buffered saline (PBS). The cells were transfected with the shRNA or plasmid at the indicated dose using Lipofectamine 3000 (L3000150) (Thermo Scientific). The cells were harvested after $48 \mathrm{~h}$ of transfection, and western blot analyses or other experiments were performed.

\section{Western blot analysis}

The proteins in cell lysates were separated by $10 \%$ sodium dodecyl sulfate-polyacrylamide gel electrophoresis (SDS-PAGE) (Bio-Rad, Hercules, CA) and electrophoretically transferred to a PVDF membrane (Amersham Pharmacia Biotech, Piscataway, NJ). The western blots were probed with specific antibodies, and the protein bands were detected using enhanced chemiluminescence.

\section{Immunohistochemistry}

The tissue array consisted of 140 formalin-fixed, paraffin-embedded (FFPE) breast cancers and corresponding adjacent normal tissues. These tissue samples were previously obtained with informed consent from patients having no anticancer treatment prior to tumor resection. The tissue specimens were histologically examined and classified according to the 2004 World Health Organization classification system. Detailed clinical and pathologic information, including the clinical and pathologic tumor-node-metastasis (TNM) stage, overall survival (OS) duration, and time to recurrence, was available for all cases. The pathological TNM status of all of the breast cancers was assessed according to the criteria of the seventh edition of the American Joint Committee on Cancer.

Immunohistochemistry was conducted using Envisionp Kit/HRP (DakoCytomation). Briefly, slides were immersed in Target Retrieval Solution ( $\mathrm{pH}$ 9; DakoCytomation) and boiled at $108^{\circ} \mathrm{C}$ for $15 \mathrm{~min}$ in an autoclave for antigen retrieval. The anti-SHMT2 antibody was added to each slide after blocking of the endogenous peroxidase and proteins, and the sections were incubated with HRP-labeled anti-rabbit IgG as the secondary antibody. The substrate-chromogen was added, and the specimens were counterstained with hematoxylin. A negative control was obtained by replacing the primary antibody with normal rabbit $\lg \mathrm{G}$.

To evaluate the immunohistochemical staining, two independent observers blinded to the clinicopathologic information performed scoring using light microscopy (magnification 20x). The intensity of SHMT2 staining was semiquantitatively evaluated using the following criteria: strongly positive (scored $2+$ ), dark-brown staining in more than $50 \%$ of the tumor cells, completely obscuring the nucleus; weakly positive (scored 1+), any lesser degree of brown staining appreciable in the tumor cell nucleus; absent (scored 0), no appreciable staining in the tumor cells. Cases were accepted as strongly positive if 2 investigators independently defined them as such. 


\section{MTT assay}

Cells plated in 96 -well plates ( 1000 cells/well) were transfected with control or specific shRNA. $48 \mathrm{~h}$ later, 10ul MTT reagent $(5 \mathrm{mg} / \mathrm{ml})$ was added in single well and treated for $2.0 \mathrm{~h}$. Then MTT was discarded and 150ul Dimethyl Sulphoxide (DMSO) was added in single well. Finally, the OD value at $490 \mathrm{~nm}$ was measured.

\section{Colony formation assay}

Cells were transfected with SHMT2 shRNA or plasmid for $24 \mathrm{~h}$, trypsinized, and resuspended as single cells. The cells $\left(1 \times 10^{3} / \mathrm{ml}\right)$ were then mixed in $21 \mathrm{ml}$ of 1640 culture miadiumr containing $5 \% \mathrm{FBS}$. The cultures were maintained in a $37^{\circ} \mathrm{C} / 5 \% \mathrm{CO} 2$ incubator for $12-14$ days. The cell clones were then washed three times with phosphate-buffered saline (PBS), fixed in methanol for 10 minutes, and stained with Crystal Violet for 10 minutes at room temperature. The dye was washed off, and the colonies that contained more than 50 cells were counted.

\section{RNA sequencing}

RNA was extracted from the vector group and the SHMT2 OVER ZR-75-1cells using the RNeasy Kit (Qiagen) according to the manufacturer's protocol. RNA quality control was performed using an Agilent Bioanalyzer. RNA-seq libraries were generated using Illumina TruSeq mRNA stranded kits following Illumina protocols. Libraries were quantitated using an Agilent bioanalyzer, and the pooled libraries were sequenced with an Illumina HiSeq 4000 system using Illumina reagents and protocols.Abundance quantifications were imported into $\mathrm{R}$ software, and gene expression matrix was constructed using $\mathrm{R}$ Bioconductor package tximport (41). Count values summarized by tximport were analyzed using the DESeq2 algorithm. Differential expression was defined at a threshold of FDR $=0.05$ and absolute log fold change $>1$.

\section{Apoptosis assay}

HCC1806 cells were inoculated in 6-well plates at a certain density and starved overnight (12 h). After cell adherence, HCC1806 cells were transfected with SHMT2 shRNA. At $48 \mathrm{~h}$ after transfection, the cells were harvested by trypsinization and fixed in 70\% cold ethanol for 30 minutes, then stained with $5 \mu$ Annexin V-FITC and $5 \mu \mathrm{PI}$ (propidium iodide) using an Annexin V-FITC/PI-staining kit (Genechem, Shanghai, China). The cells were placed at room temperature for $20 \mathrm{~min}$ in the dark and then analyzed using flow cytometry (Beckman Coulter). Apoptosis was calculated in terms of the FITC-positive cells. The raw data were analyzed using Multicycle for Windows (Beckman Coulter). 


\section{Immunofluorescence}

The treated Cells were seeded in 6 well Culture plate cover with glass slide $(24 \mathrm{mmX} 24 \mathrm{~mm})$ at a density of $1 \times 10^{5}$ cells per well. After $48 \mathrm{~h}$, the cells were washed with PBS, fixed with $4 \%$ paraformaldehyde solution, and permeabilized with $0.1 \%$ Triton X-100. The cells were incubated with a rabbit anticytochrome-c antibody and then incubated with a Goat anti-Rabbit IgG $(\mathrm{H}+\mathrm{L})$, Alexa Fluor 568 (A11036, Thermo Scientific). The nuclei were stained with 4', 6-diamidino-2-phenylindole (DAPI), and the cells were examined under a fluorescence microscope.

\section{The concentrations of VEGF and PEDF were determined by ELISA}

HCC1806 and ZR-75-1 cells were seeded in 6-well plates and treated with SHMT2 shRNA or overexpression plasmids for 48 hours. The VEGF and PEDF levels in the culture supernatant were quantified using a VEGF Immunoassay Kit (ml064281) (Enzyme-linked Biotechnology, Shanghai, Chnia) and a Chemikine PEDF ELISA Kit (ab213815) (Abcam, Shanghai, Chnia) according to the manufacturer's protocols.

\section{Animal studies}

All animal maintenance and operational procedures were carried in accordance with the animal licence protocol approval by Animal Care and Ethics Committee of Sun-Yat Sen University. Female nude mice (Balb/c) aged 5 weeks were purchased from Beijing Vital River Laboratory Animal Technology Co., Ltd. Mice were randomized for xenograft tumor growth assays. For xenograft tumor formation, the mice were randomly divided into two groups, sh-control(KD-NC), sh-shmt2(KD-SHMT2). $8 \times 10^{6}$ HCC1806 cells with stable knockdown of SHMT2 gene or control shRNA were respectively suspended in 100ul PBS and injected subcutaneously into the right flank of each mouse. The tumor volume was measured after two weeks of injection. The tumor volume was calculated as $V=$ (width $2 \times$ length) $/ 2$ and the data was recorded every three days for four weeks. Mice were sacrificed and tumors were taken from mice for weighting and photographing. Partial tissues were transferred to liquid nitrogen immediately, and partial were fixed in formalin for IHC assay.

\section{Immunohistochemistry staining assay in xenograft tumor}

The examined cancer tissues were fixed in $4 \%$ paraformaldehyde, washed with PBS three times, transferred to $70 \%$ ethanol, cut into small pieces and then embedded in paraffin in accordance with standard procedures. After being dewaxed and antigen retrieval, the tissue sections were stained using SP kit (SP-9000, ZSGB-BIO, China) according to its instructions. Briefly, the sections were blocked with blocking reagents, and then were exposed overnight at $4{ }^{\circ} \mathrm{C}$ to antibodies against SHMT2, KI67, P-P38, or 
P-ERK. The slides were washed with PBS and incubated with anti-mouse/rabbit biotin antibodies for $1 \mathrm{~h}$. After washing, the slides were added with HRP-conjugated streptavidin, developed with HRP substrate(DAB), and counterstained with hematoxylin. In the end, the paraffin sections were dehydrated by using Gradient alcohol, sealed by means of neutral balsam (Solarbio) and photographed with microscope.

\section{Statistical analysis}

All results were presented as the mean \pm SE. A Student t-test was performed to compare the two independent groups of data. A statistical analysis was performed using the SPSS statistical software package (standard version 18.0; SPSS, Chicago, IL). Survival curves were calculated using the Kaplan Meier method. The log-rank test was used to analyze overall survival (OS) time between different expression of SHMT2 group in BRCA.

\section{Results}

\section{High-expression of SHMT2 in breast cancer associated with a poor prognosis}

We first detected the expression of SHMT2 at protein levels in human normal cell lines (MCF-10A) and breast cancer cell lines (MCF-7, BT549, HCC1806, ZR-75-1) by Western blotting (Figure 1A). Among the cell lines examined, the breast cancer cell lines expressed high levels of SHMT2 protein, though the expression of SHMT2 was faint in normal breast cell lines. Western blotting was used to detect the protein expression of SHMT2 in 8 breast cancer tumor tissues, and it was also found that SHMT2 was significantly overexpressed in cancer tissues compared with matched adjacent tissues (Figure 1B).We also immunohistochemically detected the expression of the SHMT2 protein in breast cancer and adjacent area. As shown in Figure 1C, positive staining of SHMT2 was observed in breast cancer tissue but not in adjacent normal breast, suggesting that SHMT2 might be a potential biomarker of breast cancer.

We evaluated the expression of SHMT2 using TMA-containing tumors from 140 patients with breast cancer with full clinical annotation to assess the biological and clinicopathologic significance. An immunohistochemistry analysis revealed that SHMT2 was abundantly accumulated in the cytoplasm of breast cancer cells. However, SHMT2 staining can also be seen in the nuclei of some cancer cells. We classified expression on the tissue array at three levels: strongly positive (2+ score), weakly positive (1+ score), and absent (0 score). The overall survival curves revealed an unfavorable prognosis for the highSHMT2 group compared to the low-SHMT2 group (Figure 1D). Analysis of breast cancer expression profile data from TCPAC database also confirmed our findings. Compared with normal breast tissue, SHMT2 expression was significantly upregulated in cancer tissue (Figure 1E). 


\section{Up-regulation of SHMT2 promotes cell growth in breast cancers}

To assess the effects of SHMT2 on cell proliferation, we quantitatively analyzed cell viability by the MTT assay. SHMT2 knockdown by transfection with shRNA that targeting SHMT2 (sh-SHMT2) potently inhibited the proliferation of HCC1806 cells by comparison with the transfection with a non-specific scramble shRNA sequence control (sh-NC) (Figure 2A and 2B). In contrast, SHMT2 over-expression by transfection with a SHMT2-expressing vector significantly increased cell viability compared to the control group (Figure 2F and 2G).

We next analyzed the effect of SHMT2 on the changes in cell morphology. We found that cells in the control group formed a cell layer, and more spread and filopodia were observed, while SHMT2 knockdown markedly reduced cell-to-cell contact and led to a lower spreading with fewer formation of filopodia (Figure 2C). However, in the cell group of overexpressing SHMT2, it was observed that the density and total number of cells increased significantly (Figure $2 \mathrm{H}$ ).

We also performed an anchorage-independent colony formation assay to confirm the effects of SHMT2 on cancer cell growth. SHMT2 knockdown by shRNA significantly inhibited cell clonogenicity (Figure 2D and $2 \mathrm{E}$ ), resulting in a marked decrease in both the colony formation ratio. These results suggested that reductions in SHMT2 levels decrease the ability of breast cancer cells to form colonies, which was consistent with the MTT results (Figure 2B and 2G). In contrast, over-expression of SHMT2 can significantly promotes the formation of cell clones (Figure $2 \mathrm{I}$ and $2 \mathrm{~J}$ ).

\section{SHMT2 up-regulation affects multiple signaling pathways and biological processes in breast cancer cells}

In order to study the downstream signaling pathway and biological process affected by SHMT2, and to clarify the molecular mechanism of SHMT2 promoting the growth of breast cancer cells, we stably overexpressed SHMT2 in ZR-75-1 cells (Figure 2F).

Transcriptomic sequencing was used to analyze abnormal differential expression genes (DEG) in ZR-751(OVER) cells compared with ZR-75-1(VECTOR) cells (Figure 3A).RNA-seq analysis identified differentially expressed genes (DEG) in SHMT2 over-expression breast cancer cells, with differential expression defined at a threshold of false discovery rate $(F D R)=0.05$ and an absolute log2 fold change $>1.0$ (Figure 3B). A total of 3,612 differentially expressed genes were found by RNA-seq, including 1,149 up-regulated genes and 2,463 down-regulated genes (Figure 3C).Further GO analysis revealed that over-expression of SHMT2 could significantly affect multiple biological processes, including regulation of protein serine/threonine kinase activity, purine compound metabolism, immune response regulation, ubiquitinproteasome independent protein lysis process, and nucleotide metabolism, etc. (Figure 3D).In addition, KEGG enrichment analysis showed that overexpression of SHMT2 could significantly affect proteasome 
signaling pathways, ribosomal genesis, glycolysis/gluconeogenesis, serine/glycine/methionine metabolism, oxidative phosphorylation and other important signaling pathways and biochemical metabolic processes (Figure 3E).Here, overexpression of SHMT2 affects cell serine/glycine metabolism, nucleotide metabolism, immune response regulation, and ubiquitin-proteasome independent protein lysis,and all are consistent with previous literature reports(reference 123).

Next, we focused on the analysis of common signaling pathways and common intracellular biochemical metabolic processes affecting cell survival and proliferation.

We found that SHMT2 overexpression significantly affected MAPK pathway, WNT pathway, jak-STAT pathway, apoptosis pathway, VEGF and mTOR pathway (Figure 3F). In addition, SHMT2 overexpression also significantly affects intracellular oxidative phosphorylation, purine and pyrimidine metabolism, glycolysis, triglyceride metabolism, serine/glycine metabolism and RNA metabolism and other important biochemical metabolic processes (Figure $3 \mathrm{G}$ ). These results suggest that the high expression of SHMT2 in breast cancer cells is very important for cell proliferation and growth.SHMT2 can affect cell growth and proliferation in multiple paths.

\section{SHMT2 promotes breast cancer cell proliferation and growth by activating MAPK pathway}

To identify the potential molecular mechanisms by which SHMT2 knockdown inhibited breast cancer cell growth and proliferation, we analyzed the activities of several pro-survival proteins by Western blot and showed that SHMT2 knockdown dramatically suppressed the phosphorylation of ERK $1 / 2$ and p38, whereas the levels of total JNK, total ERK1/2, and total P38 protein did not change (Figure 4A). Conversely, SHMT2 over-expression increased the phosphorylation of ERK1/2 and p38, thereby leading to an activation of the MAPK signaling pathways under with over-expression of SHMT2 in breast cancer cells (Figure 4B).

To confirm the involvement of the ERK/p38 MAPK signaling pathway in the SHMT2-mediated regulation of cell growth, we analyzed the effects of the ERK and p38-selective inhibitors (FR180204 and SB202190) on SHMT2 shRNA-mediated inhibition of cell viability in breast cancer cells. Pre-treatment of the cells with FR180204 or SB202190 alone slightly inhibited cell viability. However, addition of SHMT2 shRNA did not significantly enhance the FR180204 or SB202190-mediated inhibition of cell viability (Figure 4C and 4D) due to the pre-blockage of the ERK/p38 signaling pathway by FR180204 or SB202190. On the contrary, the up-regulation of SHMT2 expression can significantly promote the growth of breast cancer cells (Figure 3D). At this point, treatment with ERK or P38 inhibitors significantly attenuated the cancer cell growth effect caused by over-expression of SHMT2 (Figure 4E and 4F). Our results indicate that SHMT2 functions partially through the modulation of the MAPK signaling to regulate breast tumor cell growth. 


\section{Knockdown of SHMT2 induces apoptosis in breast cancer cells}

We also assessed the proportion of apoptosis in HCC1806 cells transfected with shRNA sequences targeting SHMT2 (sh-SHMT2) or conrtrol shRNA (sh-NC) by Annexin V/PI staining-based FACS analysis. The knockdown of SHMT2 by shRNA resulted in a significant induction in HCC1806 cell apoptosis(Figure 5A). Conversely, apoptosis was not observed in the cells transfected with the nonspecific siRNA(Figure 5B). Because SHMT2 affects mitochondrial function and oxidative phosphorylation process. Mitochondria-mediated apoptosis pathway plays an important role in cell death process. Thus, to confirm the effect of SHMT2 knockdown on apoptosis, we next detected the expression of certain pro-apoptotic and anti-apoptotic proteins related to mitochondria mediated apoptosis pathway, cleaved-PARP, cleavedcaspase-9, cleaved-caspase-3, BAX, and Bcl-2 protein by Western blotting analysis. SHMT2 knockdown markedly induced the activation of PARP and caspase-3, resulting in an increase in the levels of cleaved PARP, cleaved caspase-3, and cleaved caspase-9 proteins. SHMT2 knockdown also increased the expression of the pro-apoptotic protein BAX and suppressed the expression of the anti-apoptotic protein BCl-2 (Figure 5C).

As cytochrome-c release is an important event in the mitochondria dependent apoptosis pathway, we next performed an immunofluorescence imaging (IF) analysis to monitor changes in the sub-cellular localization of cytochrome-c in HCC1806 cells. Transfection of cells with shRNA sequences targeting SHMT2 (sh-SHMT2) triggered the release of cytochrome-c from the inter-mitochondrial space into the cytosol by comparison with the control shRNA (sh-NC) (Figure 5D). These results suggest that blocking SHMT2 can activate mitochondrial apoptotic signaling pathways.

\section{SHMT2 regulates the VEGF/PEDF ratio in breast cells}

VEGF and PEDF protein mediated angiogenesis plays an important role in tumor growth and cancer cells proliferation $(28,29)$. We next determined the effect of SHMT2 on the expression of VEGF and PEDF proteins. Transfection with SHMT2 shRNA dramatically inhibited the expression of VEGF proteins and increased the expression of PEDF protein (Figure 6A). in contrast, SHMT2 over-expression showed an opposite effect (Figure 6B). We also examined the release of VEGF and PEDF proteins into the cell culture supernatant by ELISA. SHMT2 knockdown by shRNA significantly inhibited the release of VEGF protein but increased PEDF release in cell culture supernatant (Figure 6C and 6D). Moreover, we determined the effect of SHMT2 on the ratio of VEGF with PEDF release in cell culture supernatant. Knockdown of SHMT2 repressed the ratio of VEGF release and PEDF release in cell culture supernatant (Figure 6E). The results showed that blocking SHMT2 with SHMT2 shRNA may markedly inhibited angiogenesis related protein factor in breast cancer cells. SHMT2 may regulates the VEGF signaling pathway and influence the growth of cancer cells. 


\section{SHMT2 promotes tumor growth in vivo}

To further confirm the association between SHMT2 expression and breast cancer tumor growth, we verified the important role of SHMT2 expression in regulating breast cancer growth in vivo. HCC1806 cells with stably knockout of SHMT2 gene and the control shRNA transfection cells were suspended in 100ul PBS and subcutaneously injected into the right and left sides of each mouse's back respectively. The mice were first measured after inoculation with HCC1806 cells for one week, when the average tumor volume reached $100 \mathrm{~mm} 3$. The tumor diameters were measured at a regular interval of 4 days for up to 24 days with a digital caliper, the tumor volume and the growth curve of tumor were calculated. Knockdown of SHMT2 by shRNA significantly suppressed tumor growth (Figure 7 and 7B) and tumor weight (Figure 7C and 7D) compared to the non-specific shRNA treatment group. We also analyzed the levels of SHMT2 expression in the tumors by immunohistochemical staining and showed a significant decrease in SHMT2 expression in the tumors treated with SHMT2 shRNA (Figure 7E).

To elucidate the potential mechanisms involved in tumor growth inhibition by SHMT2 shRNA in vivo, we also analyzed the expression of ki67, p-ERK and p-p38 in tumors by immunohistochemical staining and found that $\mathrm{p}$-ERK and p-p38 expression was inhibited and the staining score of ki67 was reduced significantly (Figure 7E). These in vivo results were consistent with those observed in vitro and indicated that SHMT2 may promote tumor growth in vivo through activation of MAPK signaling pathway. SHMT2 knockdown could be a useful therapeutic strategies for breast cancer therapy.

\section{Discussion}

In this study, we evaluated the biological role, molecular mechanisms and clinical significance of SHMT2 in breast cancer carcinogenesis. As a key enzyme of cancer metabolic reprogramming, SHMT2 catalyzes the reversible reaction of serine to glycine by transferring the $\beta$-carbon from serine to tetrahydrofolate (THF). Through that reaction, serine provides the primary raw material for the de novo synthesis of purine.SHMT2 have been implicated in cancer growth, progression, metastasis, and drug resistance $(6,15$, $21,25)$. However, the biological roles and clinical significance of SHMT2 and its precise molecular mechanisms in breast cancer have not been reported.

We demonstrated the high expression of SHMT2 in breast cancer cells, tumor tissues, and breast cancer samples compared to normal cells and normal human breast tissues. To evaluate our hypothesis that SHMT2 plays a potential oncogenic role in breast cancer, we performed in vitro studies to investigate the underlying molecular mechanisms and found that SHMT2 knockdown inhibited cell viability, clonogenicity, and angiogenesis and induced apoptosis in vitro but that SHMT2 over-expression had the opposite effect. We also demonstrated the SHMT2-mediated regulation of tumor growth in a HCC1806 breast cancer xenograft mouse model in vivo.

The clinicopathologic data from our tissue array showed that patients with breast cancers, which highly expressed the SHMT2 protein, had shorter survival periods than patients with SHMT2 low expression 
tumors. These findings suggest a potential oncogenic role of SHMT2 in human breast cancer. These results are consistent with the findings of many studies that SHMT2 is expressed at high levels in a variety of cancer tissues and predicts poor prognosis(15, 21, 22, 25). These studies suggest that SHMT2 is a prognostic and diagnostic marker for cancer.

Apoptosis resistance is a protective mechanism of tumor survival and plays an important role in the response of breast cancer to chemotherapy and radiotherapy(30). In this study, we showed that the induction of apoptosis in human breast cancer cells by SHMT2 knockdown was mediated by The mitochondria-dependent apoptosis pathways. We found that SHMT2 shRNA induced the activation of caspase proteins and promoted the release of cytochrome-c from the mitochondria to cytosol. Inhibition of SHMT2 can change the balance of pro-apoptotic protein BAX and anti-apoptotic protein Bcl-2. But how does SHMT2 affect mitochondrial mediated apoptosis pathways. Whether it affects mitochondrial function directly. This is an interesting question for us to study in the future.

VEGF has been recognized as one of the principal initiators for the development and progression of vascularization, and VEGF was shown to have the most involvement both during tumor angiogenesis and also in mediating tumor cell growth and survival (31-33). PEDF counterbalances the effect of VEGF, and an increased ratio of VEGF/PEDF is required for angiogenesis and tumor growth(34). Our result also showed that SHMT2 knockdown in breast cancer cell lines led to a significant reduction in the VEGF/PEDF ratio at the protein levels, suggesting that SHMT2 at least partially targets VEGF signaling. At present, no studies have reported that SHMT2-mediated serine metabolism and one-carbon unit metabolism can affect VEGF signaling pathway and angiogenesis. Our findings suggest that SHMT2 may be involved in the regulation of tumor angiogenesis. This is a novel biological function of SHMT2. However, the molecular mechanism of SHMT2 regulating VEGF and angiogenesis needs to be further research.

In our study, we found that blocking ERK and P38 with specific inhibitors could significantly antagonize the pro-proliferation effect of SHMT2 and block the inhibitory effect of SHMT2-knockdown mediated cell proliferation. Our study suggests that SHMT2 can promote breast cancer cell proliferation by activating the MAPK pathway. It has been reported that SHMT2 can regulate AKT/MTOR signaling pathway to promote hepatocyte proliferation and regeneration(35). These results indicate that SHMT2 can regulate cell survival signaling pathway. Moreover, we demonstrated that the knockdown of SHMT2 markedly inhibited tumor growth in a breast cancer xenograft mouse model, confirming the role of SHMT2 in tumor growth and survival. Immunohistochemistry on the xenograft tumors showed that SHMT2 knockdown inhibited the survival protein factor MAPK and proliferation protein factor Ki67. Therefore, these in vivo experiments confirmed the tumor-inhibition effects by SHMT2 knockdown in vitro and provide a rationale for the pharmacologic investigation of SHMT2 as a novel therapeutic target in breast cancer cells.

\section{Conclusions}


In conclusion, we demonstrated that SHMT2 plays a critical role in human breast cancer growth by simultaneously regulating multiple signaling pathways, such as the ERK/p38 MAPK, VEGF/PEDF, and mitochondrial apoptosis pathway. Our study also demonstrated that high SHMT2 expression independently predicted a worse overall survival in patients with breast cancers. Our findings suggest that SHMT2 overexpression might help to identify BRCA patients with a poor prognosis and could therefore serve as a potential prognostic biomarker and therapeutic target for breast cancer therapy.

\section{Abbreviations}

BRCA Breast Cancer

SHMT2 Serine hydroxymethyltransferase 2

VEGF Vascular Endothelial Growth Factor

PEDF Pigment Epithelium Derived Factor

MAPK Mitogen-activated protein kinase

mTOR Mammalian target of rapamycin

PLP pyridoxal 5'-phosphate

THF tetrahydrofolate

ELISA Enzyme Linked Immunosorbent Assay

MTT 3-(4,5)-dimethylthiahiazo (-z-y1)-3,5-di- phenytetrazoliumromide

OS Overall survival

\section{Declarations}

\section{Acknowledgements:}

We apologize to the investigators whose studies we could not include due to reference number constraints. We thank Dr. Xinhua Xie from the Department of Breast Surgery, Sun-Yat sen University Cancer Center, Guangzhou, China for kindly Providing Breast cancer clinical samples and cell lines .

\section{Authors' contributions:}

Huan-Xin Lin and Ling Guo were responsible for study design and critical revision of the manuscript. Shuang-Yan Xie and Ding-bo Shi were responsible for most of the experiments, analyzed the data, prepared all the figures and wrote the manuscript. Fei Lin, Xiao-Yu Cheng, and Wen Xia provided technical 
supports. Other authors helped with the data analyses. All authors read and approved the final manuscript.

\section{Funding:}

This work was supported by the funds from the National Natural Science Foundation of China(81773103 (LHX), 82102838 (XW) and 81402254 (SDB)), Natural Science Foundation of Guangdong Province(2017A030313617 (LHX)), The Youth Innovative Talents Project of Guangdong Province ( 2018KQNCX049 (XSHY)), and the Youth scientific research project of Innovation Strong College of Guangzhou University of Chinese Medicine (2019QN09 (XSHY))

\section{Availability of supporting data:}

All data generated or analyzed during this study are included in this published article and its supplementary information files.

\section{Ethical Approval and Consent to participate:}

All animal maintenance and operational procedures were carried in accordance with the animal licence protocol approval by Animal Care and Ethics Committee of Sun-Yat Sen University Cancer Center.

\section{Consent for publication:}

All authors gave consent for the publication of the manuscript in Breast Cancer Research.

\section{Competing interests:}

The authors declare no competing financial interest.

\section{Footnotes:}

Shuang-Yan Xie and Ding-bo Shi have contributed equally to this study. Corresponding author: Ling Guo and Huan-Xin Lin

\section{References}

1. Vagia E, Mahalingam D, Cristofanilli M. The Landscape of Targeted Therapies in TNBC. Cancers (Basel) 2020;12(4). 
2. Jiang YZ, Liu Y, Xiao Y, Hu X, Jiang L, Zuo WJ, Ma D, Ding J, Zhu X, Zou J, Verschraegen C, Stover DG, Kaklamani V, Wang ZH, Shao ZM. Molecular subtyping and genomic profiling expand precision medicine in refractory metastatic triple-negative breast cancer: the FUTURE trial. Cell Research 2021;31(2):178-86.

3. Zhao S, Zuo WJ, Shao ZM, Jiang YZ. Molecular subtypes and precision treatment of triple-negative breast cancer. Ann Transl Med 2020;8(7):499.

4. Geeraerts SL, Heylen E, De Keersmaecker K, Kampen KR. The ins and outs of serine and glycine metabolism in cancer. Nat Metab 2021;3(2):131-41.

5. Li AM, Ye J. Reprogramming of serine, glycine and one-carbon metabolism in cancer. Biochim Biophys Acta Mol Basis Dis 2020;1866(10):165841.

6. Xie M, Pei DS. Serine hydroxymethyltransferase 2: a novel target for human cancer therapy. Invest New Drugs 2021.

7. Zeng Y, Zhang J, Xu M, Chen F, Zi R, Yue J, Zhang Y, Chen N, Chin YE. Roles of Mitochondrial Serine Hydroxymethyltransferase 2 (SHMT2) in Human Carcinogenesis. Journal of Cancer 2021;12(19):5888-94.

8. Yang L, Garcia CJ, Chen Z, Wang L, Liang L, Jang C, Mayr JA, Zhang Z, Ghergurovich JM, Zhan L, Joshi S, Hu Z, McReynolds MR, Su X, White E, Morscher RJ, Rabinowitz JD. Serine Catabolism Feeds NADH when Respiration Is Impaired. Cell Metabolism 2020;31(4):809-21.

9. Ye J, Fan J, Venneti S, Wan YW, Pawel BR, Zhang J, Finley LW, Lu C, Lindsten T, Cross JR, Qing G, Liu Z, Simon MC, Rabinowitz JD, Thompson CB. Serine catabolism regulates mitochondrial redox control during hypoxia. Cancer Discovery 2014;4(12):1406-17.

10. Tramonti A, Cuyas E, Encinar JA, Pietzke M, Paone A, Verdura S, Arbusa A, Martin-Castillo B, Giardina G, Joven J, Vazquez A, Contestabile R, Cutruzzola F, Menendez JA. Metformin Is a Pyridoxal-5'phosphate (PLP)-Competitive Inhibitor of SHMT2. Cancers (Basel) 2021;13(16).

11. Morscher RJ, Ducker GS, Li SH, Mayer JA, Gitai Z, Sperl W, Rabinowitz JD. Mitochondrial translation requires folate-dependent tRNA methylation. Nature 2018;554(7690):128-32.

12. Pikman Y, Ocasio-Martinez N, Alexe G, Dimitrov B, Kitara S, Diehl FF, Robichaud AL, Conway AS, Ross L, Su A, Ling F, Qi J, Roti G, Lewis CA, Puissant A, Vander HM, Stegmaier K. Targeting serine hydroxymethyltransferases 1 and 2 for T-cell acute lymphoblastic leukemia therapy. Leukemia 2021.

13. Parsa S, Ortega-Molina A, Ying HY, Jiang M, Teater M, Wang J, Zhao C, Reznik E, Pasion JP, Kuo D, Mohan P, Wang S, Camarillo JM, Thomas PM, Jain N, Garcia-Bermudez J, Cho BK, Tam W, Kelleher NL, Socci N, Dogan A, De Stanchina E, Ciriello G, Green MR, Li S, Birsoy K, Melnick AM, Wendel HG. The serine hydroxymethyltransferase-2 (SHMT2) initiates lymphoma development through epigenetic tumor suppressor silencing. Nat Cancer 2020;1:653-64.

14. Ducker GS, Ghergurovich JM, Mainolfi N, Suri V, Jeong SK, Hsin-Jung LS, Friedman A, Manfredi MG, Gitai Z, Kim H, Rabinowitz JD. Human SHMT inhibitors reveal defective glycine import as a targetable metabolic vulnerability of diffuse large B-cell lymphoma. Proc Natl Acad Sci U S A 2017;114(43):11404-9. 
15. Nguyen TH, Vemu PL, Hoy GE, Boudjadi S, Chatterjee B, Shern JF, Khan J, Sun W, Barr FG. Serine hydroxymethyltransferase 2 expression promotes tumorigenesis in rhabdomyosarcoma with 12q13q14 amplification. Journal of Clinical Investigation 2021;131(15).

16. Liu C, Wang L, Liu X, Tan Y, Tao L, Xiao Y, Deng P, Wang H, Deng Q, Lin Y, Jie H, Zhang H, Zhang J, Peng Y, Zhang H, Zhou Z, Sun Q, Cen X, Zhao Y. Cytoplasmic SHMT2 drives the progression and metastasis of colorectal cancer by inhibiting beta-catenin degradation. Theranostics 2021;11(6):2966-86.

17. Wei Z, Song J, Wang G, Cui X, Zheng J, Tang Y, Chen X, Li J, Cui L, Liu CY, Yu W. Deacetylation of serine hydroxymethyl-transferase 2 by SIRT3 promotes colorectal carcinogenesis. Nature Communications 2018;9(1):4468.

18. Chen J, Na R, Xiao C, Wang X, Wang Y, Yan D, Song G, Liu X, Chen J, Lu H, Chen C, Tang H, Zhuang G, Fan G, Peng Z. The loss of SHMT2 mediates 5-fluorouracil chemoresistance in colorectal cancer by upregulating autophagy. Oncogene 2021;40(23):3974-88.

19. DeNicola GM, Chen PH, Mullarky E, Sudderth JA, Hu Z, Wu D, Tang H, Xie Y, Asara JM, Huffman KE, Wistuba II, Minna JD, DeBerardinis RJ, Cantley LC. NRF2 regulates serine biosynthesis in non-small cell lung cancer. Nature Genetics 2015;47(12):1475-81.

20. Tong J, Krieger JR, Taylor P, Bagshaw R, Kang J, Jeedigunta S, Wybenga-Groot LE, Zhang W, Badr H, Mirhadi S, Pham NA, Coyaud E, Yu M, Li M, Cabanero M, Raught B, Maynes JT, Hawkins C, Tsao MS, Moran MF. Cancer proteome and metabolite changes linked to SHMT2. PLoS One 2020;15(9):e237981.

21. Luo L, Zheng Y, Lin Z, Li X, Li X, Li M, Cui L, Luo H. Identification of SHMT2 as a Potential Prognostic Biomarker and Correlating with Immune Infiltrates in Lung Adenocarcinoma. Journal of Immunology Research 2021;2021:6647122.

22. Ji L, Tang Y, Pang X, Zhang Y. Increased Expression of Serine Hydroxymethyltransferase 2 (SHMT2) is a Negative Prognostic Marker in Patients with Hepatocellular Carcinoma and is Associated with Proliferation of HepG2 Cells. Med Sci Monit 2019;25:5823-32.

23. Zeng Y, Zhang J, Xu M, Chen F, Zi R, Yue J, Zhang Y, Chen N, Chin YE. Roles of Mitochondrial Serine Hydroxymethyltransferase 2 (SHMT2) in Human Carcinogenesis. Journal of Cancer 2021;12(19):5888-94.

24. Yang X, Wang Z, Li X, Liu B, Liu M, Liu L, Chen S, Ren M, Wang Y, Yu M, Wang B, Zou J, Zhu WG, Yin Y, Gu W, Luo J. SHMT2 Desuccinylation by SIRT 5 Drives Cancer Cell Proliferation. Cancer Research 2018;78(2):372-86.

25. Zhang L, Chen Z, Xue D, Zhang Q, Liu X, Luh F, Hong L, Zhang H, Pan F, Liu Y, Chu P, Zheng S, Lou G, Yen Y. Prognostic and therapeutic value of mitochondrial serine hydroxyl-methyltransferase 2 as a breast cancer biomarker. Oncology Reports 2016;36(5):2489-500.

26. Li AM, Ducker GS, Li Y, Seoane JA, Xiao Y, Melemenidis S, Zhou Y, Liu L, Vanharanta S, Graves EE, Rankin EB, Curtis C, Massague J, Rabinowitz JD, Thompson CB, Ye J. Metabolic Profiling Reveals a 
Dependency of Human Metastatic Breast Cancer on Mitochondrial Serine and One-Carbon Unit Metabolism. Molecular Cancer Research 2020;18(4):599-611.

27. Bernhardt S, Bayerlova M, Vetter M, Wachter A, Mitra D, Hanf V, Lantzsch T, Uleer C, Peschel S, John J, Buchmann J, Weigert E, Burrig KF, Thomssen C, Korf U, Beissbarth T, Wiemann S, Kantelhardt EJ. Proteomic profiling of breast cancer metabolism identifies SHMT2 and ASCT2 as prognostic factors. Breast Cancer Research 2017;19(1):112.

28. Han Z, Dong Y, Lu J, Yang F, Zheng Y, Yang H. Role of hypoxia in inhibiting dendritic cells by VEGF signaling in tumor microenvironments: mechanism and application. American Journal of Cancer Research 2021;11(8):3777-93.

29. Becerra SP, Notario V. The effects of PEDF on cancer biology: mechanisms of action and therapeutic potential. Nature Reviews Cancer 2013;13(4):258-71.

30. Saatci O, Kaymak A, Raza U, Ersan PG, Akbulut O, Banister CE, Sikirzhytski V, Tokat UM, Aykut G, Ansari SA, Dogan HT, Dogan M, Jandaghi P, Isik A, Gundogdu F, Kosemehmetoglu K, Dizdar O, Aksoy S, Akyol A, Uner A, Buckhaults PJ, Riazalhosseini Y, Sahin O. Targeting lysyl oxidase (LOX) overcomes chemotherapy resistance in triple negative breast cancer. Nature Communications 2020;11(1):2416.

31. Kong D, Zhou H, Neelakantan D, Hughes CJ, Hsu JY, Srinivasan RR, Lewis MT, Ford HL. VEGF-C mediates tumor growth and metastasis through promoting EMT-epithelial breast cancer cell crosstalk. Oncogene 2021;40(5):964-79.

32. Song Y, Zeng S, Zheng G, Chen D, Li P, Yang M, Luo K, Yin J, Gu Y, Zhang Z, Jia X, Qiu N, He Z, Li H, Liu H. FOXO3a-driven miRNA signatures suppresses VEGF-A/NRP1 signaling and breast cancer metastasis. Oncogene 2021;40(4):777-90.

33. Zhang YQ, Chen WL, Zhang F, Wei XL, Zeng D, Liang YK, Wu JD, Zhang LY, Guo CP, Zeng HC, Hao SS, Li RH, Huang WH, Zhang GJ. Over-expression of both VEGF-C and Twist predicts poor prognosis in human breast cancer. Clinical \& Translational Oncology 2019;21(9):1250-9.

34. Rivera-Perez J, Monter-Vera M, Barrientos-Alvarado C, Toscano-Garibay JD, Cuesta-Mejias T, FloresEstrada J. Evaluation of VEGF and PEDF in prostate cancer: A preliminary study in serum and biopsies. Oncology Letters 2018;15(1):1072-8.

35. Wang M, Yuan F, Bai H, Zhang J, Wu H, Zheng K, Zhang W, Miao M, Gong J. SHMT2 Promotes Liver Regeneration Through Glycine-activated Akt/mTOR Pathway. Transplantation 2019;103(7):e188-97.

\section{Figures}


A.

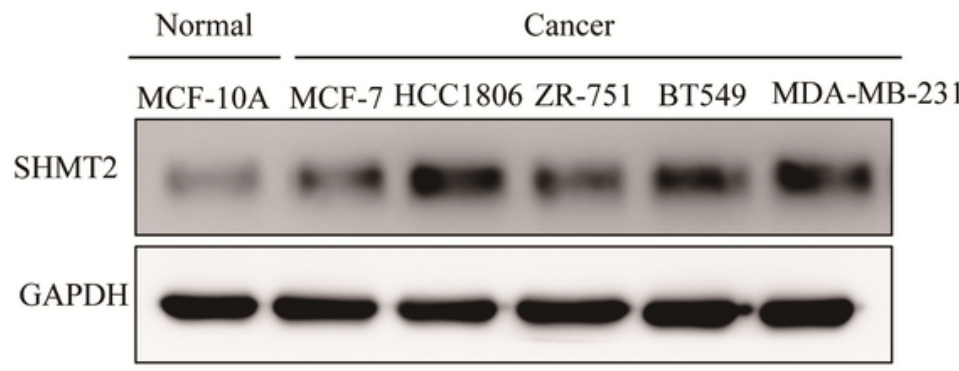

B.

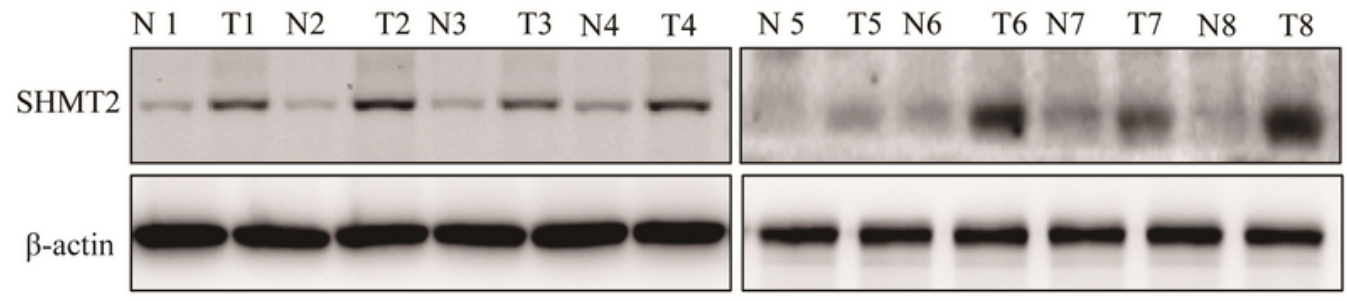

C.

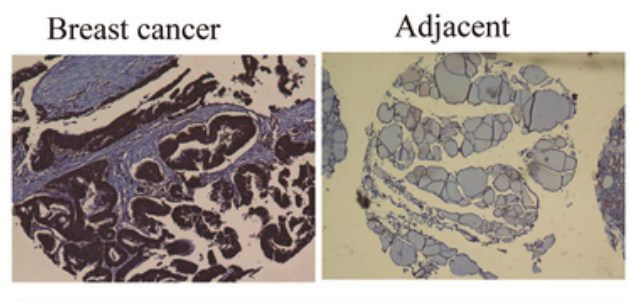

Case 1

D.

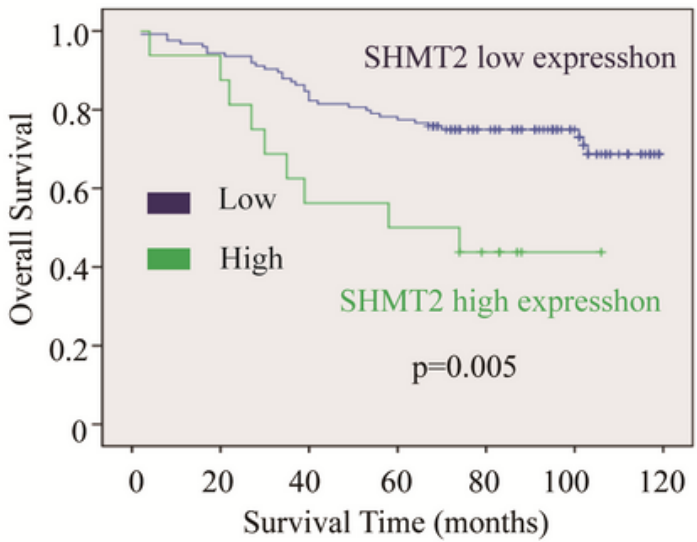

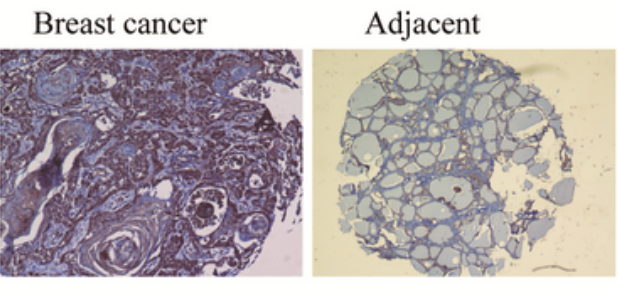

Case 2

E.

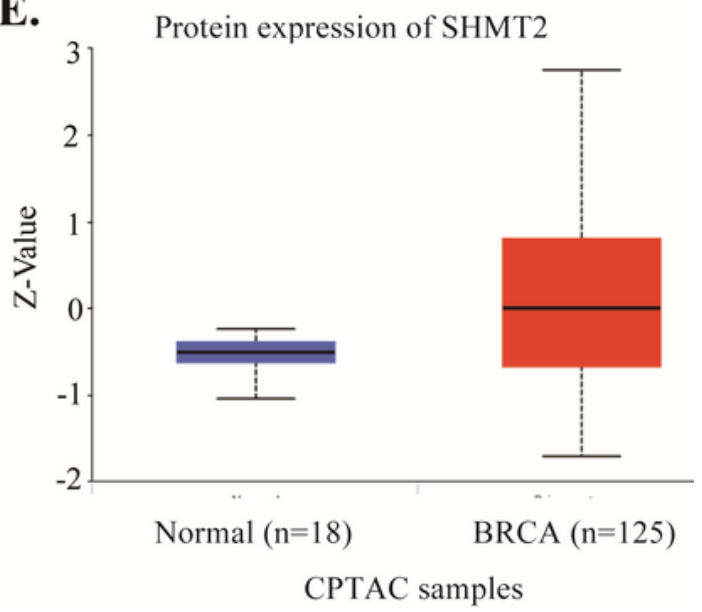

\section{Figure 1}

SHMT2 protein is highly expressed in breast cancer cells and tumor tissues and associated with a poor prognosis. (A) Expression of SHMT2 proteins in various Breast cancer cell lines (MCF-1,MDA-MB231,HCC1806,ZR-75-1,BT549) and a nomal breat cell line (mcf-10A)were analyzed by Western blotting using a human SHMT2 antibody. (B) Expression of SHMT2 proteins were analyzed by Western blotting in eight cases of breast cancer tissue and matched paracancer tissue . T,breast cancer tissue,N,matched 
paracancer tissue. (C)Strong staining of SHMT2 was detected by immunohistochemistry in representative breast cancer tissues and matched paracancer tissues. A strong positive staining was observed in cancer cells, whereas the adjacent area staining was weak. Original magnification, 200x. (D) SHMT2 overexpression correlates with a poor prognosis in patients with breast cancers. Kaplan-Meier curves of overall survival in all patients $(n=140)$. (E) Analysis of CPTAC gene expression profile data showed that SHMT2 was highly expressed in breast cancer tissues compared with normal breast tissues.

A.

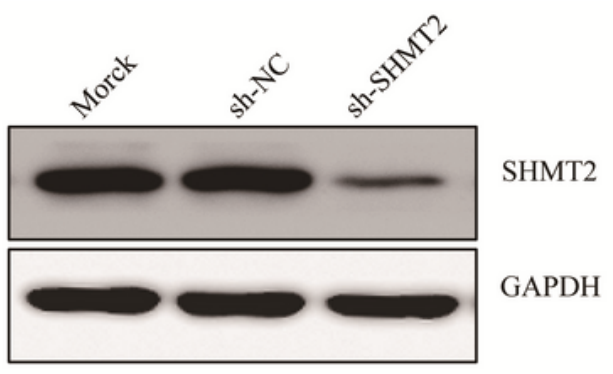

B.

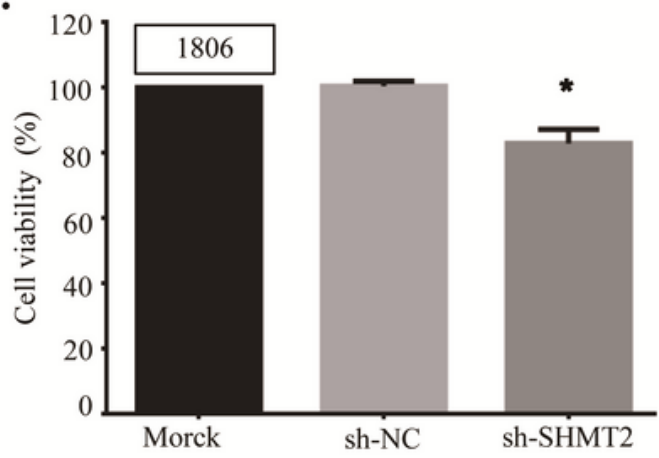

C.

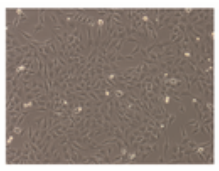

Morck

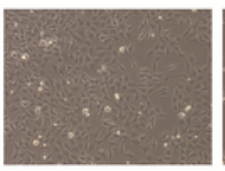

sh-NC
F.

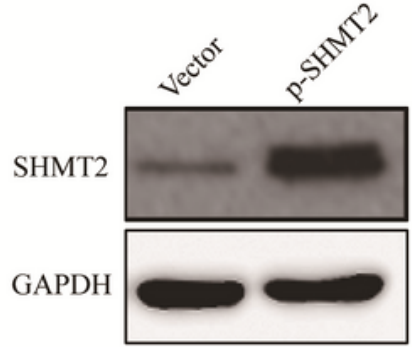

G.

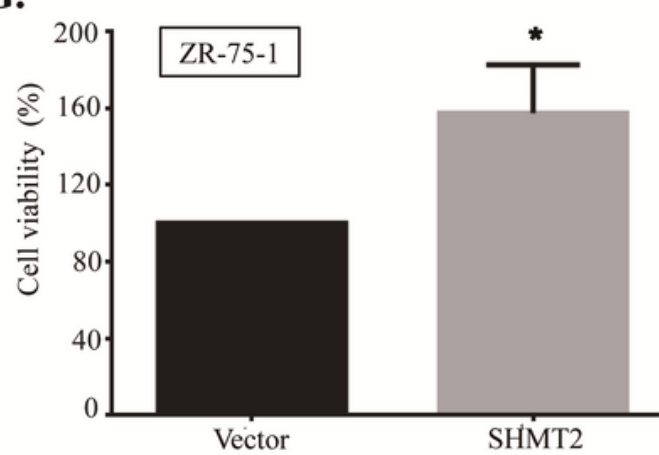

H.

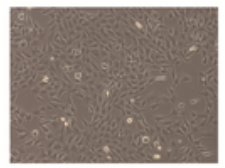

Vector

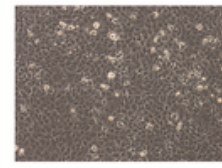

SHMT2

I.

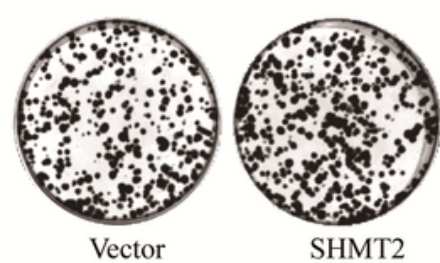

J.

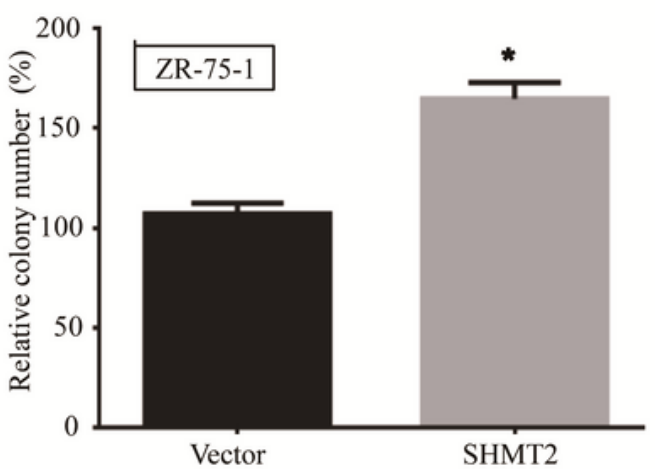

Figure 2 
High expression of SHMT2 promotes breast cell growth in vitro. The expression of SHMT2 in breast cancer cell line HCC1806 (A) and ZR-75-1 (F) was detected by immunoblotting technique. GAPDH detection as internal reference. The breast cancer cell line HCC1806 was transfected with SHMT2 interfering RNA (SH-SHMT2) and control RNA for two days. The ZR-75-1 cells were transfected with empty control vector and SHMT2 overexpressed plasmid (P-SHMT2) for two days. The cell viability was determined by an MTT assay in breast cancer cell line HCC1806 (B) and ZR-75-1 cells(G) at 48 hours after transfection respectivily. Cells treated with DMSO were used as the reference group(MorcK), with the cell viability set at $100 \%$. The percent cell viability in each treatment group was calculated relative to the cells treated with the vehicle control. The HCC1806 (C) and ZR-75-1 cells $(\mathrm{H})$ were photographed after transfection. The tumor cells HCC1806 (D) and ZR-75-1 (I) induced colony formation was also analyzed by the plate clone formation experiment under with knock down or up-regulation of SHMT2 respectivily. The relativily colony formation rate were calculated in HCC1806 (D) and ZR-75-1 cells (J) trated with knock down or up-regulation of SHMT2 respectively.The data are presented as the mean \pm SD of three tests. ${ }^{*} P<0.05$, significant differences between the treatment groups and control groups. sh-NC means non-specific shRNA control.Vector group means the control group of SHMT2 Overexpresstion group. 
A.

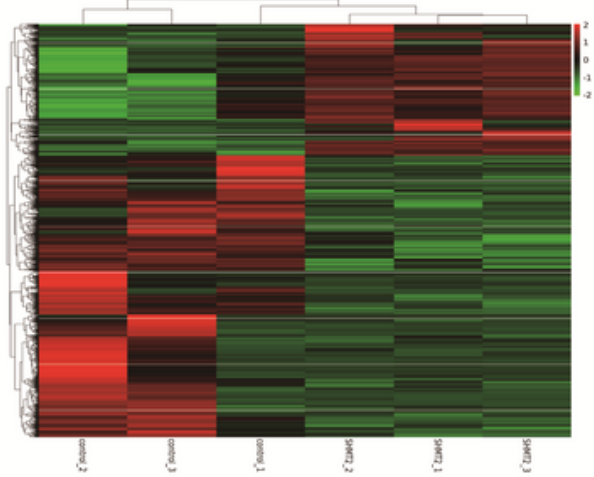

C.

D.
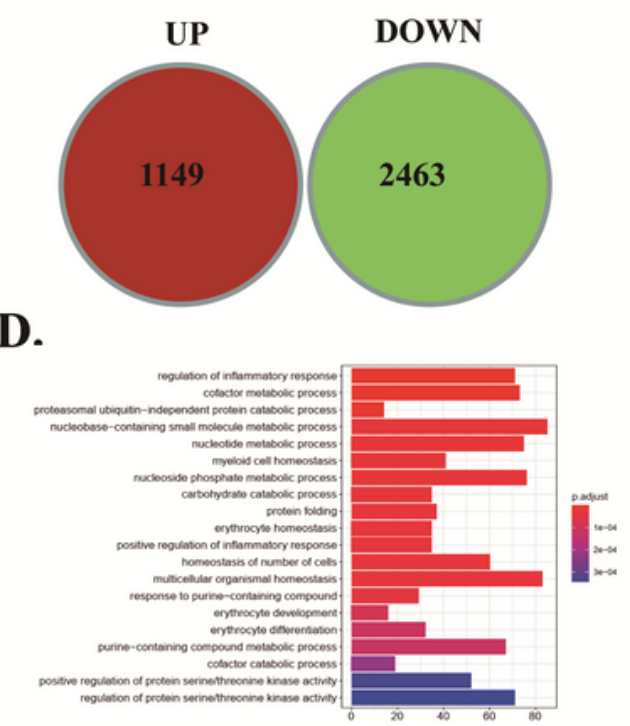

F.

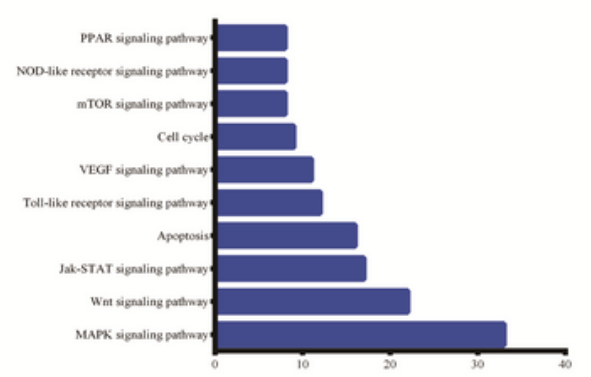

B.

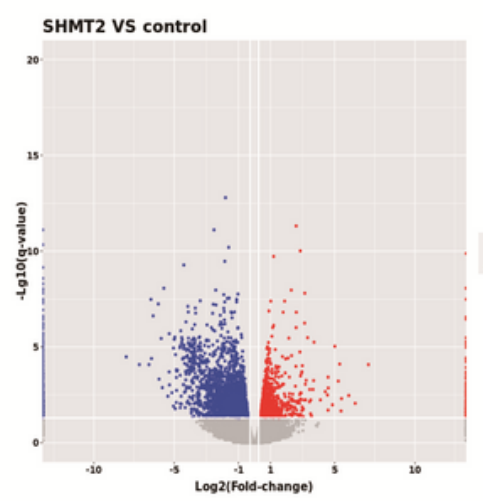

E.

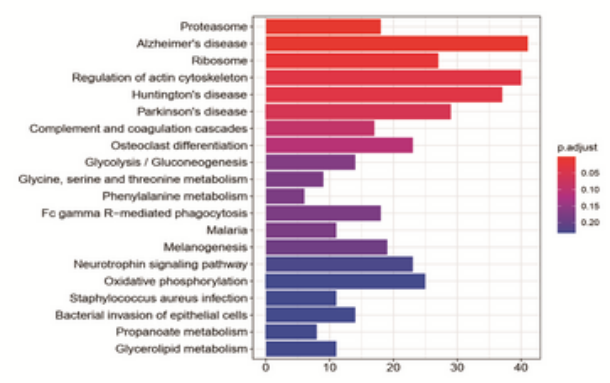

G.

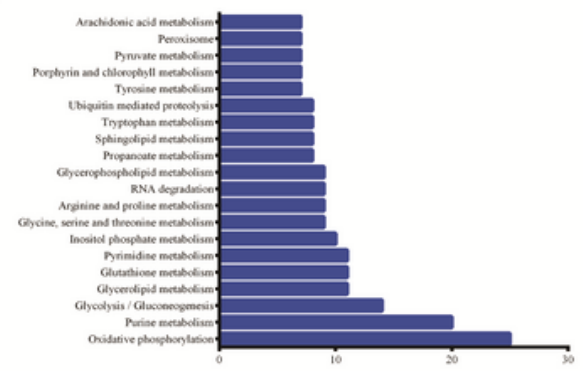

\section{Figure 3}

SHMT2 up-regulation affects multiple signaling pathways and biological processes in breast cancer cells.Stably over-expressed SHMT2 protein in ZR-75-1 cells. Transcriptomic sequencing was used to analyze abnormal differential expression genes (DEG) in ZR-75-1(over) cells compared with ZR-751 (vector) cells $(\mathrm{N}=3)$.(A)Clustering results of differentially expressed genes in SHMT2 overexpression cells compaired with the control group cells.(B)Volcanic map of differentially expressed mrnas.(C)RNA-seq 
analysis identified differentially expressed genes (DEG) in SHMT2 over-expression breast cancer cells. A total of 3,612 differentially expressed genes were found by RNA-seq, including 1,149 up-regulated genes and 2,463 down-regulated genes.(D)GO enrichment histogram of differentially expressed mRNA.(E)KEGG enrichment bars of differentially expressed mRNA.(F)Common signaling pathways closely associated with cell proliferation and survival.(G)SHMT2 overexpression affects a variety of biochemical metabolic processes in breast cancer cells.

A.

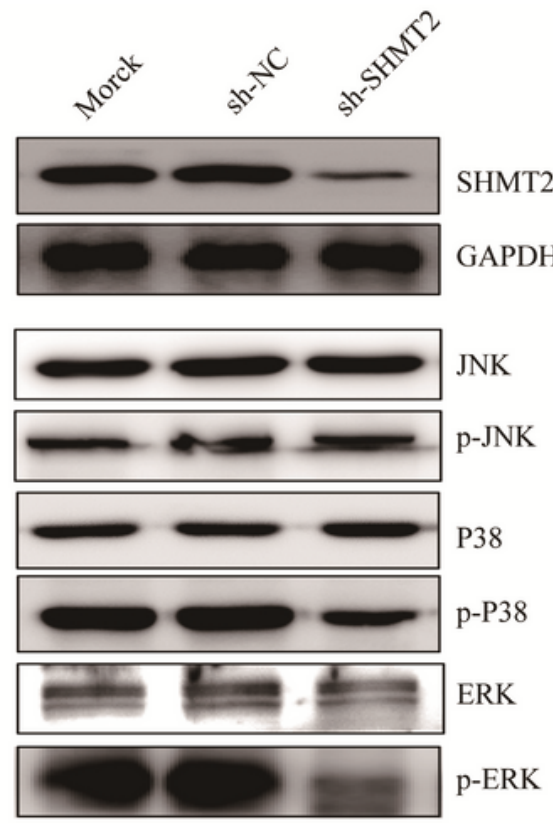

C.

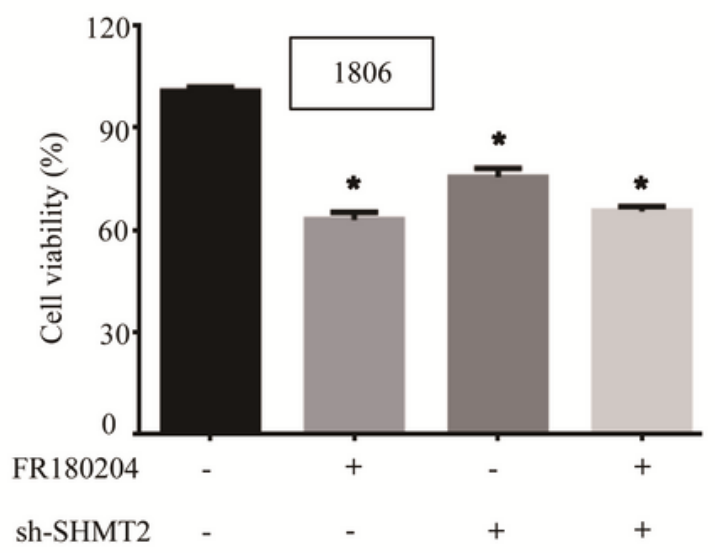

E.

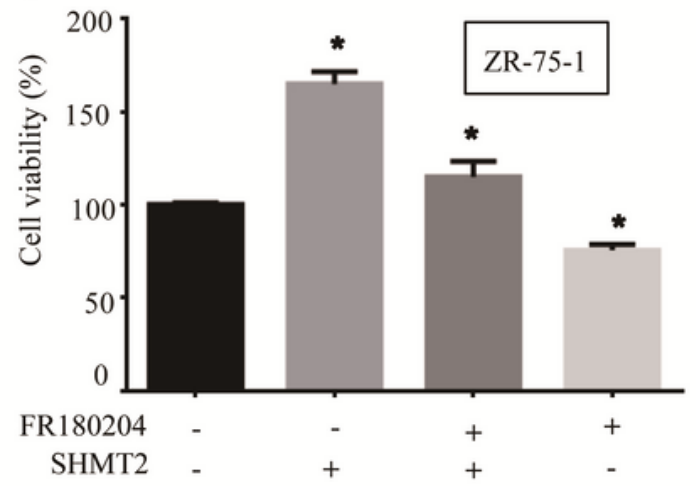

B.
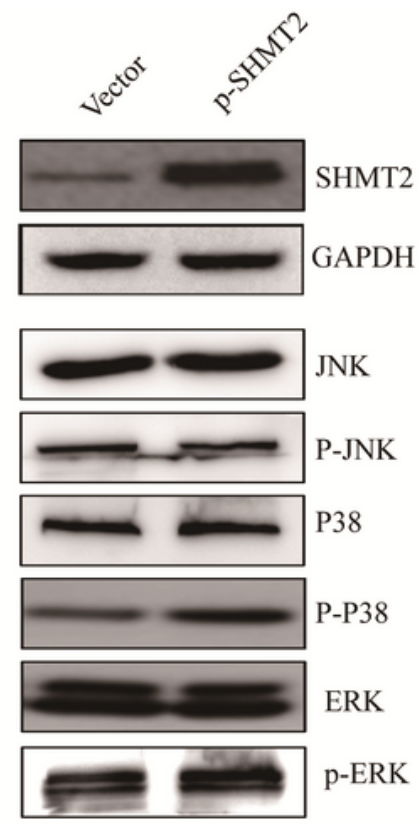

D.

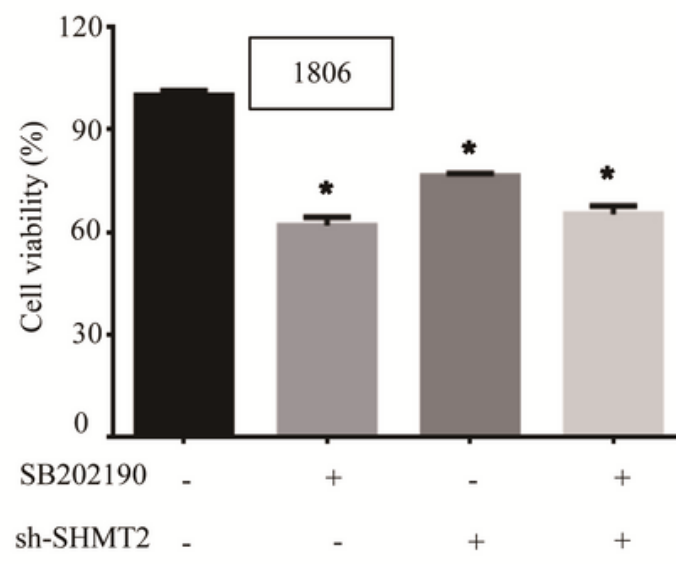

F.

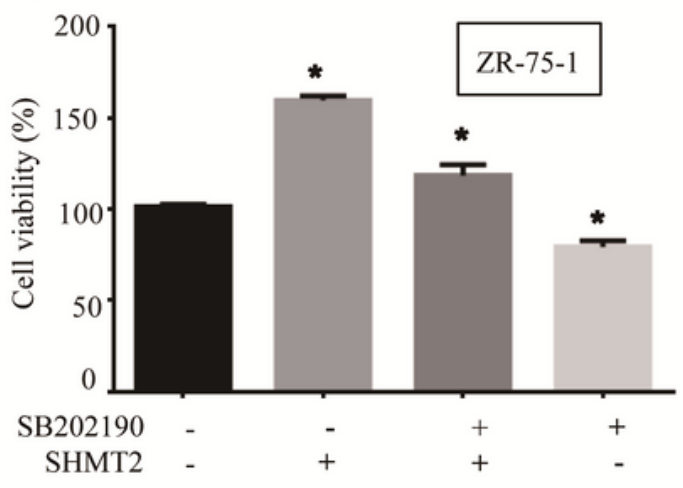

Figure 4 
SHMT2 promotes breast cancer cell proliferation and growth by activating MAPK pathway Human HCC1806 cells were transfected with SHMT2 shRNA (sh-SHMT2) or the control shRNA (NC-SHMT2)(A), and the ZR-75-1 cells were transfected SHMT2-expressing plasmids or the control vector plasmid respectively (B). At 48 hours after transfection, the levels of SHMT2, total and phosphorylated JNK, p38 and ERK proteins were analyzed by Western blotting. HCC1806 cells were pre-treated with an ERK inhibitor FR180204 (C) or a p38 inhibitor SB202190 (D) respectively in a certain concentration for $4 \mathrm{~h}$, then transfected cells with SHMT2 shRNA or the control shRNA plasimd respectively, After $48 \mathrm{~h}$, cell viability was determined by MTT analysis. ZR-75-1 cells were pre-treated with an ERK inhibitor FR180204 (E) or a p38 inhibitor SB202190 (F) in a certain concentration for $4 \mathrm{~h}$, and then transfected cells with the control vector plasmid or over-expression plasimd of SHMT2 respectively, After $48 \mathrm{~h}$, cell viability was determined by MTT analysis. ${ }^{\star} \mathrm{P}<0.05$, significant differences between the treatment groups and control group. 
A.
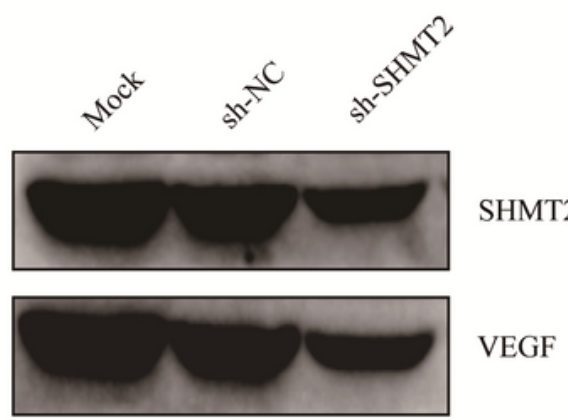

VEGF

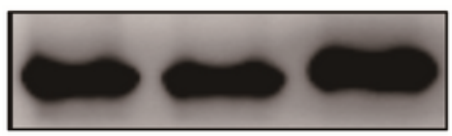

PEDF

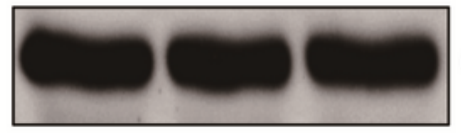

GAPDH

C.

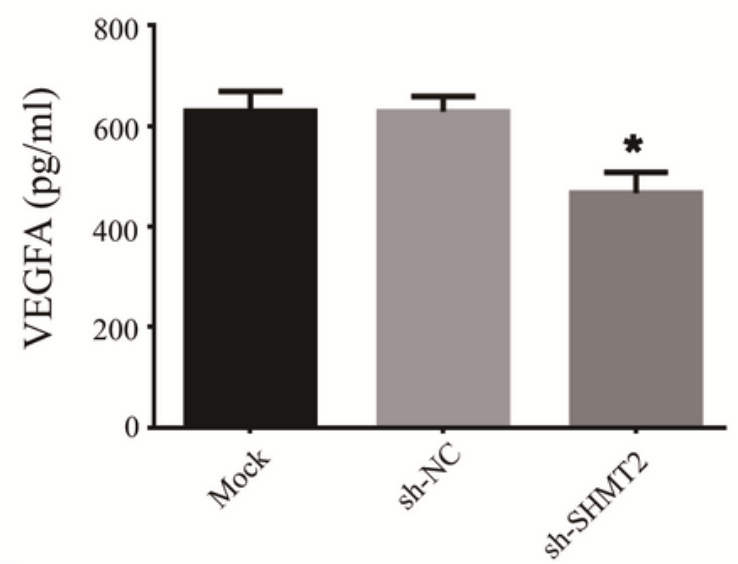

E.

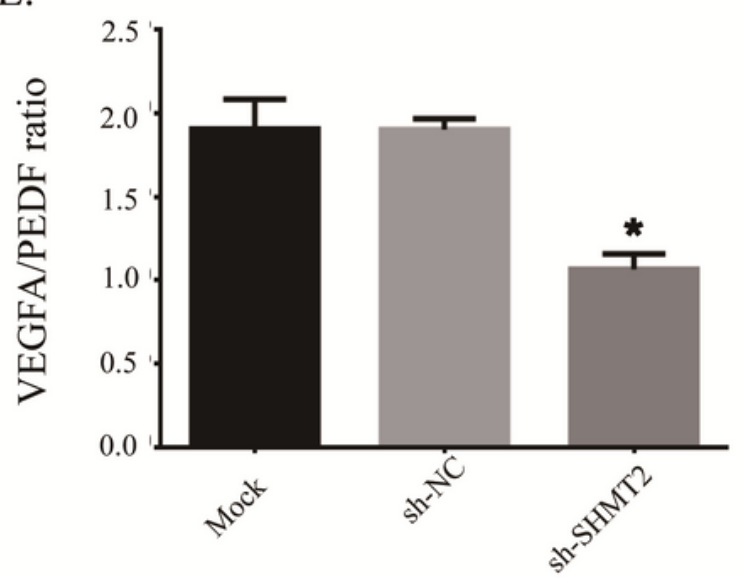

B.
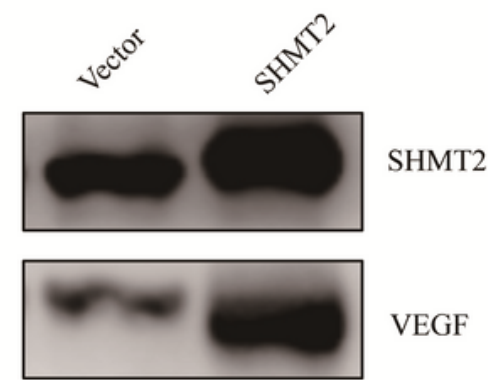

VEGF

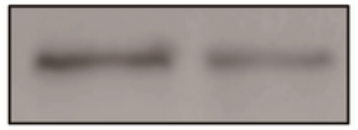

PEDF

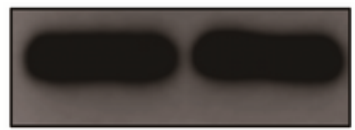

GAPDH

D.

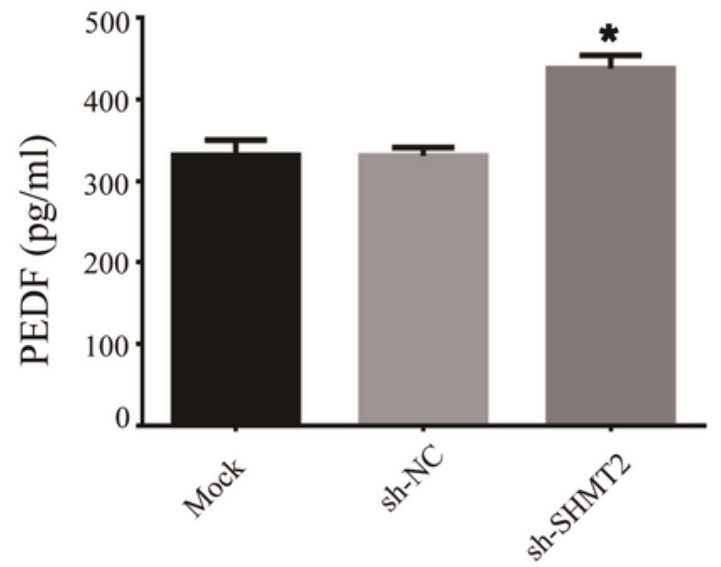

\section{Figure 5}

knockdown of SHMT2 activates caspase dependent apoptotic pathway. (A)HCC1806 cells were inoculated with 6-well plates according to certain density and starved for 24 hours, and were transfected with RNA sequences targeting SHMT2 (sh-SHMT2). At 48 hours after transfection, Apoptosis rate was detected by annexinv-FITC and PI double stained cells combined with flow cytometry fluorescence separation.(B)The apoptosis rate of each group was quantitatively analyzed.Apoptosis is represented by 
the relative percentage of apoptotic cells versus that of the contro group cells. ${ }^{*} P<0.05$, significant differences between the treatment groups and Mock groups.(C)The levels of SHMT2,cleaved PARP, cleaved caspase-3, cleaved caspase-9, BAX, and Bcl-2 proteins were analyzed by western blotting . GAPDH detection as a loading control. (D)The release of cytochrome-c was analyzed by an immunofluorescence imaging analysis to monitor cytochrome-c release from the inter-mitochondrial space into the cytosol .DAPI staining for nuclei.

A.

Mock

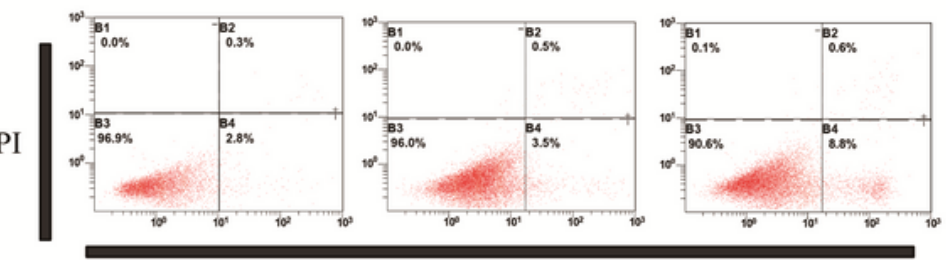

FITC

B.

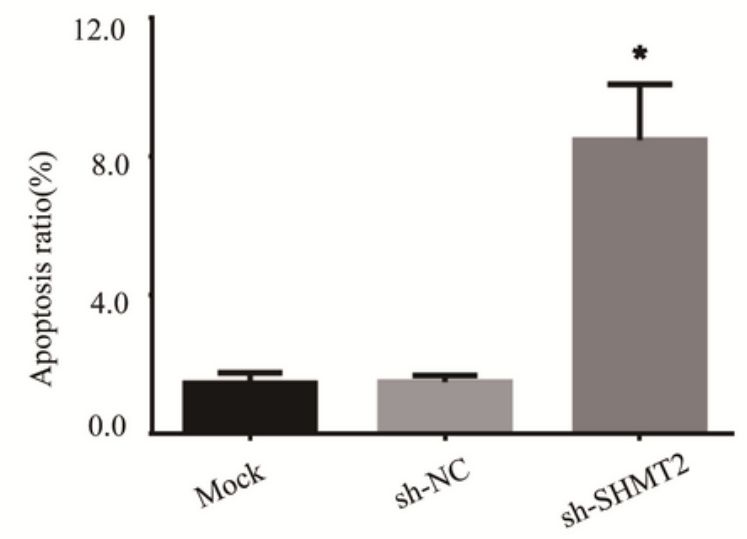

D.
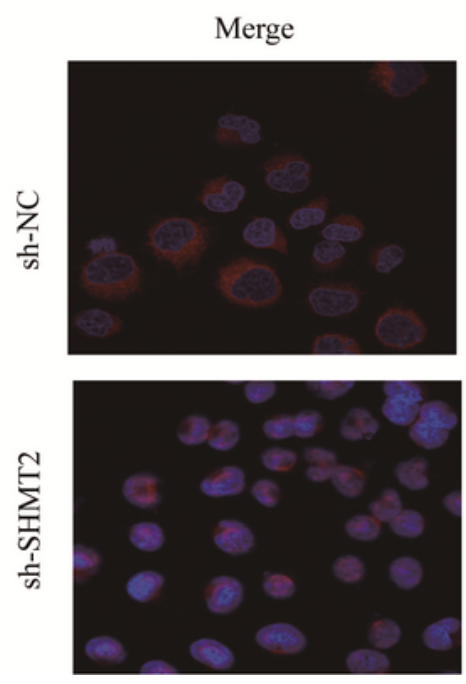

C.

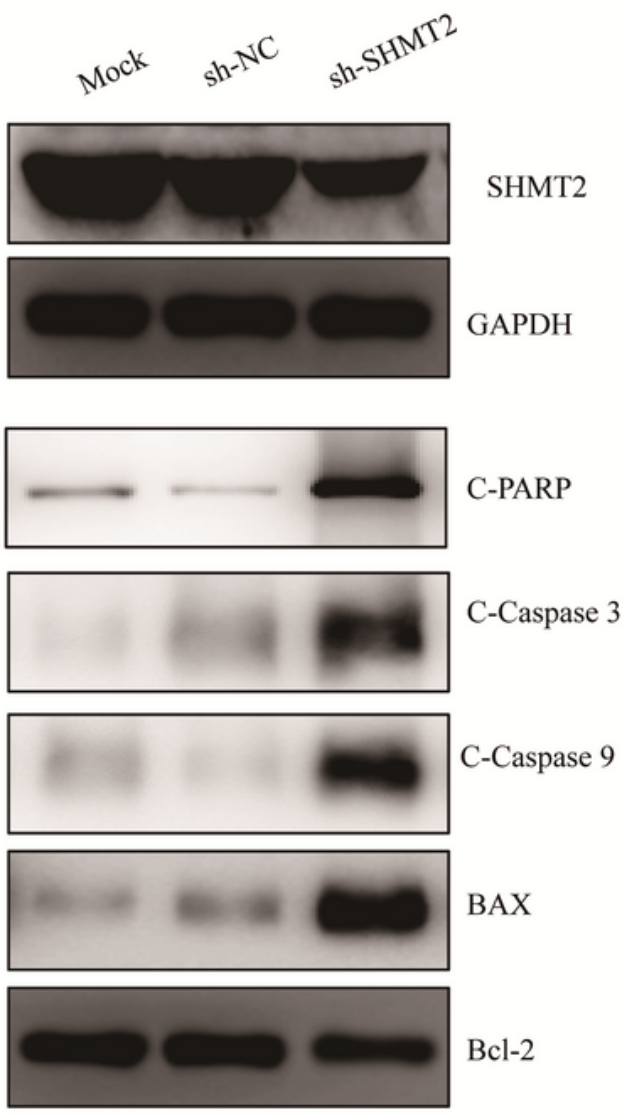

DAPI
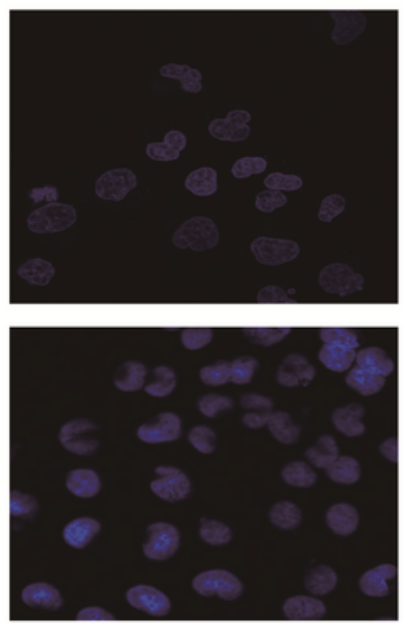

Figure 6 
knockdown of SHMT2 inhibits VEGF signaling pathway. (A) Human HCC1806 cells were transfected with SHMT2 shRNA (sh-SHMT2) or the overexpression plasmid of SHMT2 (p-SHMT2) (B). At 48 hours after transfection, the effects of SHMT2 knockdown or overexpression on the expression of SHMT2, VEGF and PEDF at protein levels were detected by Western blotting, respectively. GAPDH detection as a loading control. (C)The amount of VEGF and PEDF proteins released (D) in cell to the culture supernatant were determined by ELISA in the HCC1806 cells knock down to SHMT2 or overexpresstion of SHMT2 respectivily. (E) the relative ratio of VEGF protein release to PEDF protein release was analyzed in the cell culture supernatant in $\mathrm{HCC} 1806$. The data are presented as the mean \pm SD of three separate experiments. ${ }^{*} \mathrm{P}<0.05$, significant differences between the treatment groups and control groups. 
A.

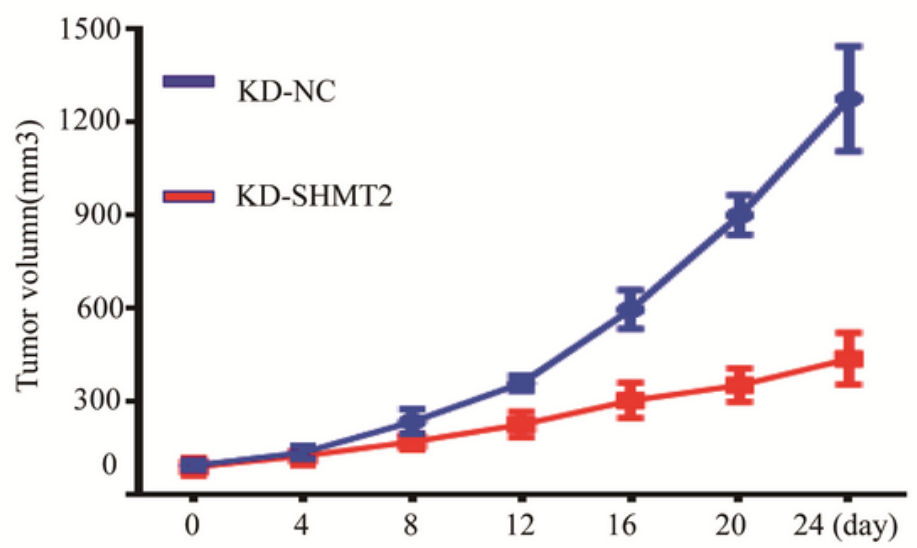

C.

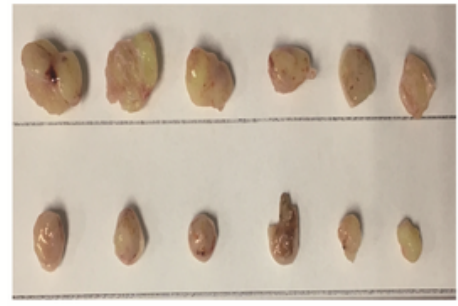

E.

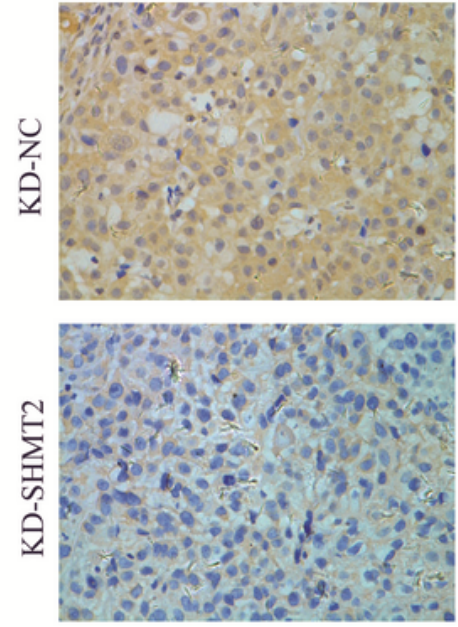

p-ERK
KD-NC

KD-SHMT2
L: KD-NC

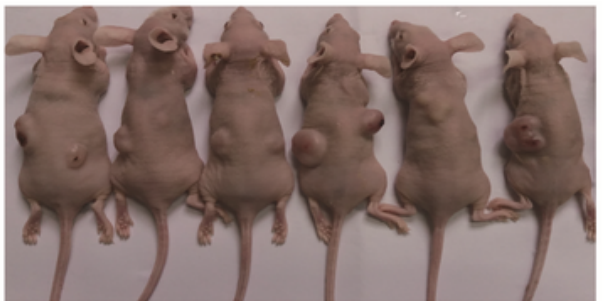

D.

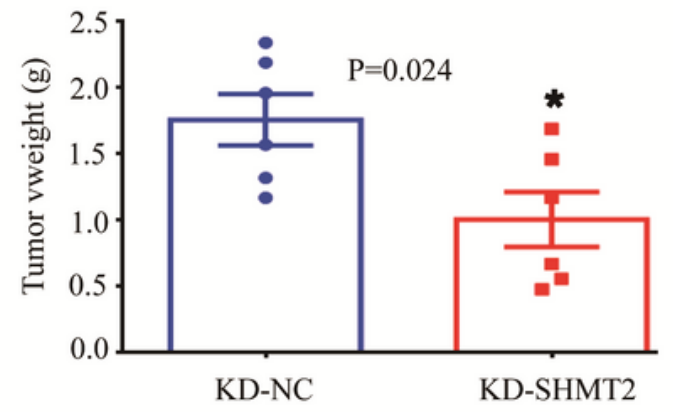

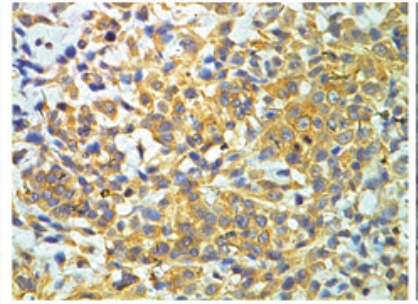
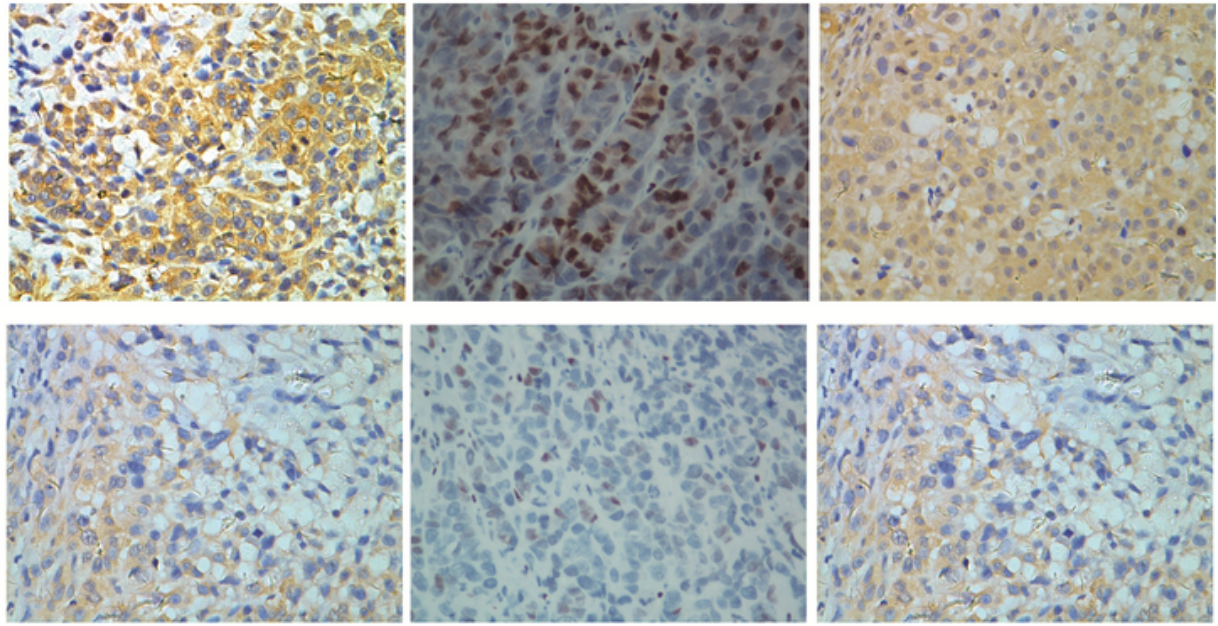

p-p38

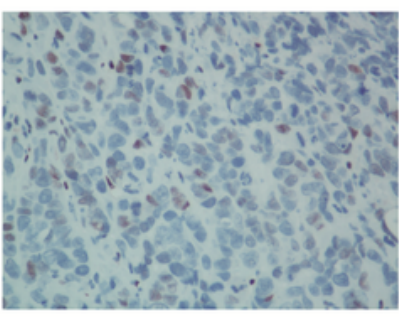

KI67

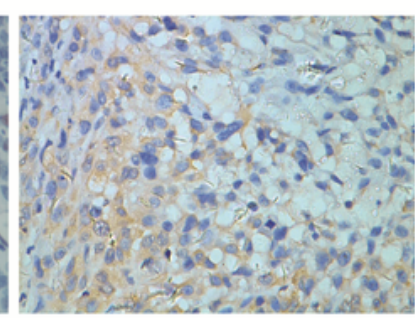

SHMT2

\section{Figure 7}

Knockdown of SHMT2 inhibits breast tumor growth in vivo. HCC1806 cells stably knockout of SHMT2 gene and the control shRNA transfection cells were suspended in 100ul PBS and subcutaneously injected into the right and left sides of each mouse's back respectively. The mice were first measured after inoculation with HCC1806 cells for one week, when the average tumor volume reached $100 \mathrm{~mm}$. The tumor diameters were measured at a regular interval of 4 days for up to 24 days with a digital caliper, the 
tumor volume and the growth curve of tumor were calculated (A). The mice were killed and photographed after seven measurements (B). The xenograft was removed and photographed (C), and the weights of the xenografts were analyzed (D). The expression levels of p-ERK, p-p38, ki67 and SHMT2 proteins in tumor tissues were detected by immunohistochemical staining $(E) . P<0.05$, significant differences between the SHMT2 shRNA groups and non-specific scramble shRNA groups. $\mathrm{n}=5$ mice/group, Magnification, $200 \times$ 